\title{
In-silico Testing of a Generalized Predictive Control Based Artificial Pancreas for Child Patients with Type 1 Diabetes
}

\author{
Wenping Liu ${ }^{1}$, Ting Chen ${ }^{1}$, Liling Yu ${ }^{1}$ and Haoyu Jin ${ }^{1+}$ \\ ${ }^{1}$ Institute of Medical Devices, Guangdong Food and Drug Vocational College, Guangzhou, Guangdong, \\ China
}

\begin{abstract}
We have developed a generalized predictive control (GPC) based controller with two adaptive strategies, namely, an adaptive reference glucose trajectory (ARGT) and an adaptive softening factor (ASF), for artificial pancreas systems (AP). Tests with the UVA/Padova type 1 diabetes mellitus simulator (T1DMS), approved by the US Food and Drug Administration, showed that it realized the effective control of the blood glucose concentrations (BGCs) of adult and adolescent patients. Here, the GPC controller and those two adaptive strategies (ARGT and ASF) were further tested with 10 in-silico children by using the UVA/Padova T1DMS. Results showed that ARGT and ASF strategies significantly increased the quality of the GPC controller and the BGCs of 10 child patients were effectively controlled with the GPC+ARGT+ASF controller.
\end{abstract}

Keywords: generalized predictive control, artificial pancreas, children, adaptive softening factor, adaptive reference glucose trajectory

\section{Introduction}

Type 1 diabetes (T1D) is a chronic autoimmune disease characterized by deficiency of insulin production in the body. It is generally caused by the autoimmune destruction of pancreatic beta cells, resulted from a complex interplay between environmental factors and microbiome, genome, metabolism, and immune systems[1, 2].

The artificial pancreas (AP) system, or the closed-loop control system, is a promising approach to maintain blood glucose concentrations (BGCs) of T1D patients in a euglycemic range (70-180 mg/dl or 3.89-10 mmol/L) [3-5]. The world's first AP system, Medtronic's MiniMed 670G system, was approved by the US Food and Drug Administration (FDA) in 2016. The AP system consists of three types of devices, namely, continuous glucose monitoring (CGM), intelligent controller and insulin-infusion pump (Fig. 1a). The real-time CGM system provides the patient's blood glucose levels, while the intelligent controller calculates the optimum amount of insulin to be infused with an insulin infusion pump.

At present, several control algorithms have been applied to the intelligent controller of AP systems, including proportional-integral-derivative (PID) control, model predictive control (MPC) and generalized predictive control (GPC) et al. [6-12]. GPC has received a lot of attention in the past few years because it is fairly robust and can be easily implemented [13-15]. We have developed a GPC controller with two strategies, namely, an adaptive reference glucose trajectory (ARGT) and an adaptive softening factor (ASF). Its performance was tested by using the UVA/Padova type 1 diabetes mellitus simulator (T1DMS) approved by the US FDA, involving 10 adults and 10 adolescents. Test results showed that it realized the effective control of BGCs of those 20 in-silico subjects [16]. Here, four GPC controller, including the traditional GPC controller, the GPC+ARGT controller, the GPC+ASF controller and the GPC+ARGT+ASF controller, were tested with 10 in-silico children by using the UVA/Padova T1DMS. Results showed the ARGT and ASF strategies significantly increased the quality of the GPC controller. The BGC percentages within the

\footnotetext{
+ Corresponding author. Tel.: + 0086-20-29164611; fax: + 0086-20-29164611.

E-mail address: jinhy@gdyzy.edu.cn.
} 
euglycemic range of 10 child subjects was $79.03 \%$ with the GPC+ARGT+ASF controller. It is comparable with several other controllers tested with T1DMS. For example, the BGC percentage within the euglycemic range of in-silico subjects with the PIDD, PID and MPC controllers was $77 \%, 72.6 \%$ and $79.6 \%$, respectively $[10,11]$. Therefore, the GPC+ARGT+ASF controller was effective and can be potentially applied in the AP systems.
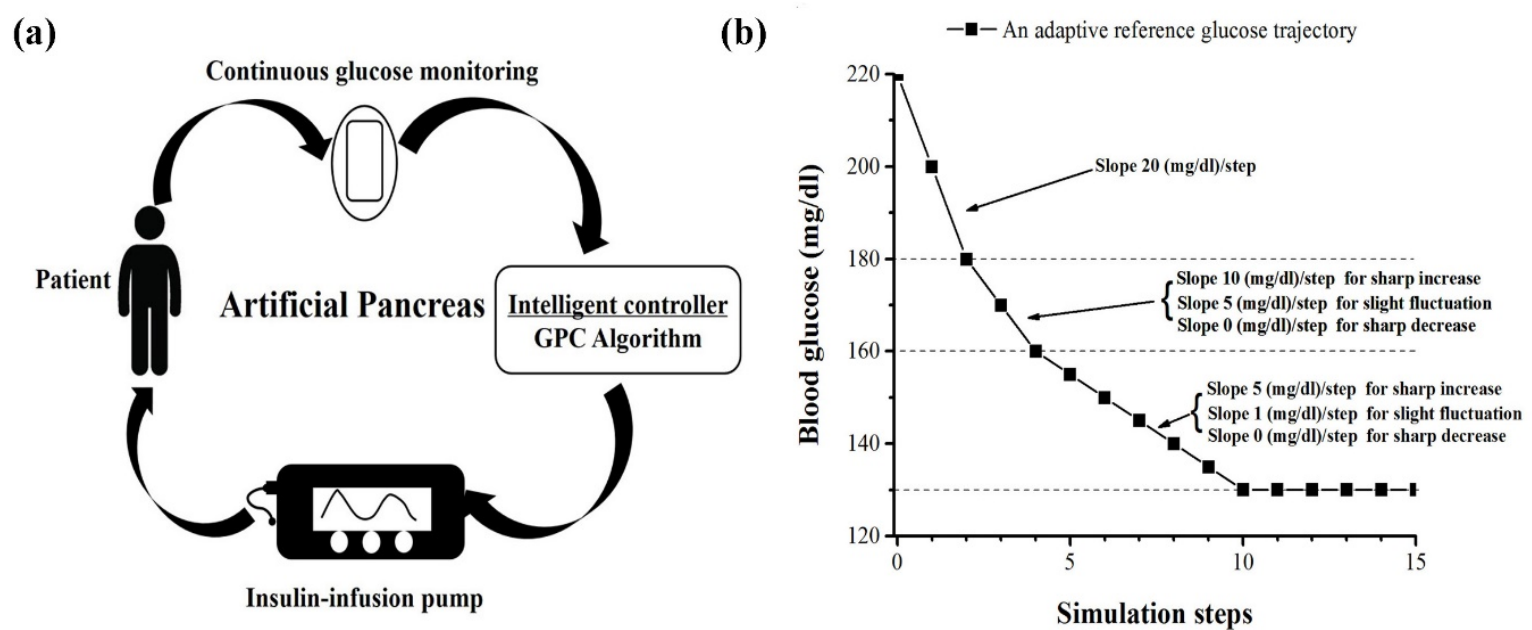

Fig. 1: Sketch map of an artificial pancreatic system (a) and adaptive reference glucose trajectory (b).

\section{Methods}

\subsection{GPC Algorithm}

A GPC controller is designed and applied for blood glucose regulation in our previous research[16]. Insulin injection rate is computed by minimizing $\mathrm{J}$ in the following function.

$$
\begin{gathered}
\mathrm{J}=\sum_{j=1}^{n}[y(k+j)-w(k+j)]^{2}+\sum_{j=1}^{m} \lambda(j)[\Delta u(k+j-1)]^{2} \\
w(k+j)=\alpha^{j} y(k)+\left(1-\alpha^{j}\right) y_{r} \quad(j=1,2, \ldots, n)
\end{gathered}
$$

Where $y(k+j)$ represents the $j$-step-ahead prediction of the process output. It is estimated using an autoregressive integrated moving-average model with exogenous inputs (function 2) and the Diophantine equation. $\Delta u(k+j-1)$ donates the incremental control input at the $k$ th sampling step. $n$, denoting the output prediction horizon. $m$ denotes the control horizon. $\lambda(j)$, denoting the weighting parameter toward deviation of the control input. $y(k)$ denotes the current process output, and $y_{r}$ denotes the reference glucose trajectory. $\alpha$ denotes the weight of $y(k)$, also known as softening factor. The minimization of function (1) gives the optimal control action (insulin infusion rate) and its solution process is introduced in our previous research in detail [16].

$$
\begin{gathered}
\mathrm{A}\left(z^{-1}\right) y(k)=B\left(z^{-1}\right) u(k-1)+C\left(z^{-1}\right) \xi(k) / \Delta \\
\mathrm{A}\left(z^{-1}\right)=1+a_{1} z^{-1}+\cdots+a_{n a^{-} z^{-n a}} \\
\mathrm{~B}\left(z^{-1}\right)=b_{0}+b_{1} z^{-1}+\cdots+b_{n b^{2}} z^{-n b} \\
\mathrm{C}\left(z^{-1}\right)=1+c_{1} z^{-1}+\cdots+c_{n c^{-n}} z^{-n c}
\end{gathered}
$$

\subsection{ARGT}

The ARGT was proposed in our previous research, instead of a desired glucose value or a time-varying trajectory [9, 17]. It avoids the sudden decrease of BGCs and increases the effectiveness of the GPC controller for patient subjects. The slopes of the trajectory in Fig. 1b were adjusted in real time in accordance with the increasing (or decreasing) BGC rates measured in the past two steps.

\subsection{ASF}

The softening factor $(\alpha)$ can vary from 0 to 1 and significantly affects the integral time and output of the GPC system. When $\alpha$ is set to 0 , the GPC system will likely have low robustness and high tracking speed; when $\alpha$ is set to 1 , it will have high robustness and slow tracking speed. An ASF strategy was proposed to ensure the balance between track speed and system robustness. $\alpha$ is calculated in real time with the BGC variations in the past two steps, that is, 


$$
\alpha=m^{-\left|y_{k}-y_{k-1}\right|}
$$

where $m=1.1, y_{k}$ and $y_{k-1}$ denote the BGCs measured in the past two steps.

\subsection{Software and Parameter Setting}

The UVA/Padova T1DMS version 3.2.1 was used to build and test the GPC controllers. It embodies the biophysiological parameters of the FDA-accepted in-silico populations, including 10 adults, 10 adolescents and 10 children [18-20]. The mean age and weight of the adult group is 34.80 and 72.91(kg), respectively. The mean age and weight of the adolescent group is 17.60 and $51.17(\mathrm{~kg})$, respectively. The mean age and weight of the child group is 5.10 and $30.60(\mathrm{~kg})$, respectively.

The scenario was demonstrated as below[16]. Each test was run for 3 days (72 hours), and each child was provided 3 meals a day. The multiple meals given during the testing period were as follows: $30 \mathrm{~g} \mathrm{CHO}$ at 7 a.m., $30 \mathrm{~g}$ at 12 p.m., and $30 \mathrm{~g}$ at 6 p.m. daily (i.e., at the 7th, 12th, 18th, 31st, 36th, 42nd, 55th, 60th, and 66th hour).

\section{Results and Discussion}

The performance of four GPC controllers was evaluated here, including the traditional GPC controller, the GPC+ARGT controller, the GPC+ASF controller and the GPC+ARGT+ASF controller. Those four GPC controllers were proposed in our previous research and tested with 10 adults and 10 adolescents. Here, they were further tested with 10 child patients.

The percentages of 10 child patients with BGCs within the euglycemic region were used to verify the effectiveness of the GPC controllers. The BGC percentages of 10 children for the without-GPC-controller scenario (no insulin injection) was 27.25\%, 26.24\%, 30.76\%, 10.65\%, 58.20\%, 46.77\%, 30.83\%, 26.75\%, $22.29 \%$ and $25.76 \%$, respectively. Fig. 2 showed their merged BGC density. The average BGC percentage within the euglycemic region was only $30.54 \% \pm 13.16 \%$ (average \pm standard deviation), indicating abnormally high levels of blood glucose.

\subsection{In-silico Testing of the Traditional GPC Controller}

Firstly, the traditional GPC algorithm were tested with 10 child patients. Its design was clearly described in our previous research, which adopted a time-varying trajectory and a fixed softening factor value of 0.6 [16].

The BGC percentages of 10 subjects (child 01, 02, 03, 04, 05, 06, 07, 08, 09 and 10) increased by 49.13\%, 34.67\%, 38.69\%, 47.48\%, 11.95\%, 44.25\%, 27.12\%, 45.78\%, 24.78\% and 50.17\%, respectively, in relation to their results without GPC controller. The merged BGC density of 10 children was shown in Fig. 2. The average BGC percentage within the euglycemic region was $67.94 \% \pm 12.36 \%$. Although it was considerably higher than that without GPC controllers (30.54\%), the performance of the traditional GPC controller was not satisfactory.

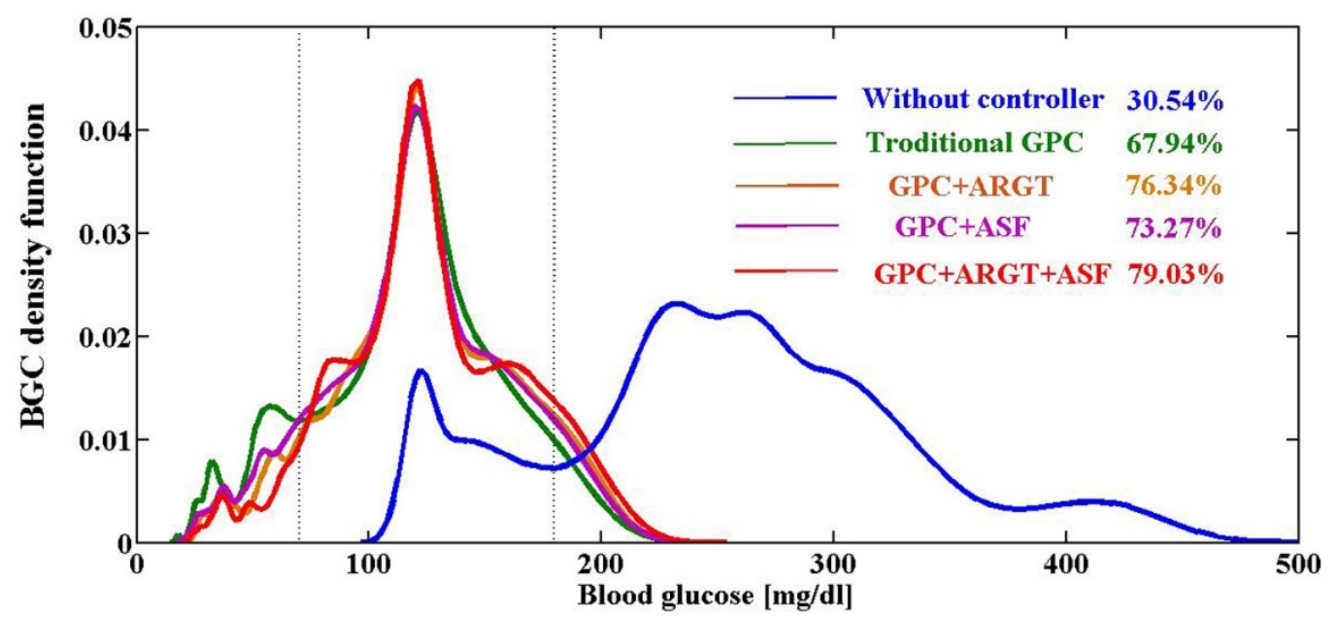

Fig. 2: Merged BGC density of 10 child patients without controller or with different GPC controllers. The BGC percentages within the euglycemic region are labelled. Black dotted lines represent the euglycemic region. 


\subsection{In-silico Testing of the GPC+ARGT Controller}

Then, tests of the GPC+ARGT controller was performed with 10 children. In relation to the 10 subjects in the GPC algorithm test in Section 3.1, the BGC percentages of 10 subjects increased by 3.33\%, 6.99\%, $10.69 \%, 3.18 \%, 7.22 \%, 1.3 \%, 13.47 \%, 15.64 \%, 2.34 \%$ and 19.86\%, respectively. The merged BGC density of 10 children was shown in Fig. 2. The average BGC percentage within the euglycemic region of the patient subjects was $76.34 \% \pm 14.32 \%$, and it was higher than that in Section $3.1(67.94 \% \pm 12.36 \%)$. F-tests showed that the variance of the BGC percentages of 10 subjects with GPC+ARGT controller was equal to that in Section $3.1(\mathrm{~F}=0.74, \mathrm{p}=0.33)$. Then, T-tests were performed to further examine the difference. But it did not reveal significant differences between the two patient-groups (Fig. 3).

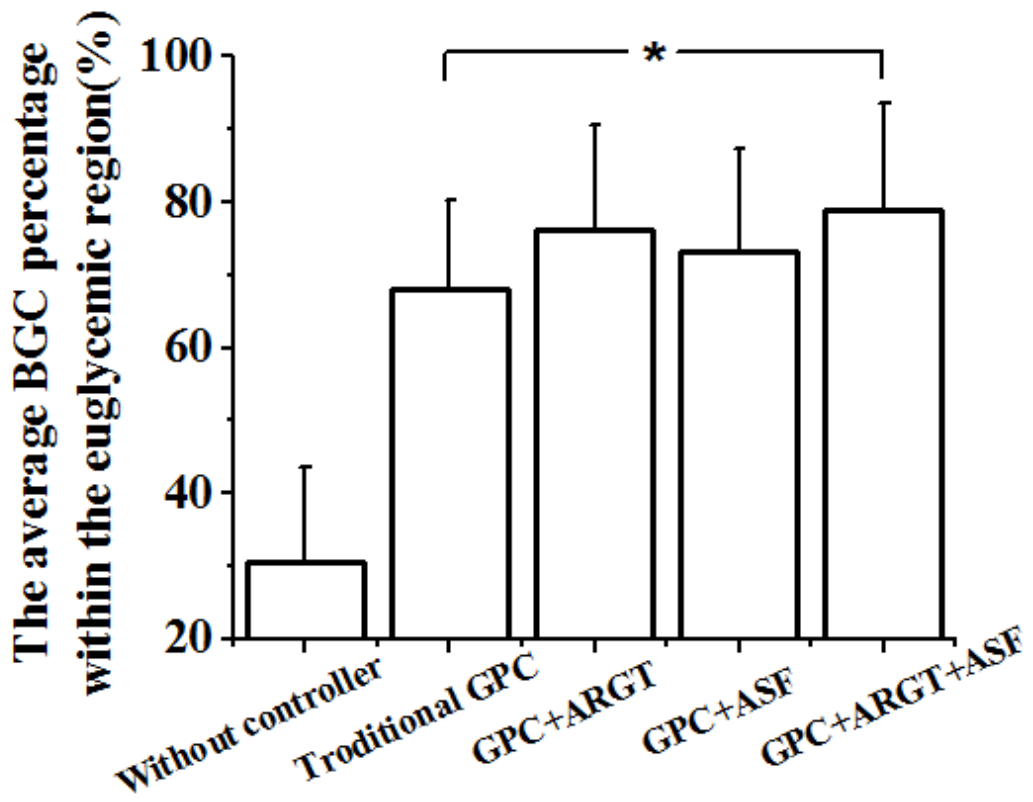

Fig. 3: T-test of BGC percentages within the euglycemic region with different GPC controllers. * $\mathrm{p}<0.05$.

\subsection{In-silico Testing of the GPC+ASF Controller}

The effectiveness of the GPC+ASF controller was also evaluated with the UVA/Padova T1DMS. In relation to the 10 subjects in the GPC algorithm test in Section 3.1, the BGC percentages of 10 subjects increased by $9.86 \%, 5.56 \%, 7.18 \%, 2.46 \%, 7.12 \%, 2.11 \%, 0.44 \%, 11.13 \%, 1.88 \%$ and $5.58 \%$, respectively. The merged BGC density of 10 subjects was shown in Fig. 2. The average BGC percentage within the euglycemic region of the patient group was $73.27 \% \pm 14.08 \%$. Although it was higher than that in Section $3.1(67.94 \% \pm 12.36 \%)$, following F-tests and T-tests did not reveal significant differences between the two patient-groups (Fig.3)

\subsection{In-silico Testing of the GPC+ARGT+ASF Controller}

At last, the GPC+ARGT+ASF controller was tested with 10 child patients. In relation to the GPC algorithm test in Section 3.1, the BGC percentages of 9 subjects (subjects 01, 02, 03, 04, 05, 07, 08, 09 and 10) increased by $14.21 \%, 9.19 \%, 13.15 \%, 2.46 \%, 12.03 \%, 33.12 \%, 19.46 \%, 1.88 \%$ and $7.18 \%$, respectively, whereas one remaining subject (subjects 06) decreased slightly by $1.83 \%$. The merged BGC density of 10 subjects was shown in Fig.2. The average BGC percentage within the euglycemic regions of the patient subjects was $79.03 \% \pm 14.57 \%$, and it was higher than that in Section 3.1 (67.94\% $\pm 12.36 \%)$. F-tests showed that the variance of the BGC percentages of 10 subjects with GPC+ARGT+ASF controller was equal to that in Section $3.1(\mathrm{~F}=0.72, \mathrm{p}=0.32)$. Thus, $\mathrm{T}$-tests were performed to further examine the difference. Consequently, the average BGC percentage within the euglycemic region for the patient group (79.03\% $\pm 14.57 \%$ ) was significantly higher than that shown in Section 3.1 (67.94\% $\pm 12.36 \%$ ) (Fig.3). Therefore, the ARGT and ASF strategies significantly increased the quality of the GPC controller. The GPC+ARGT+ASF controller is effective for child patients with T1D and can be potentially applied in the AP systems. 


\section{Conclusions}

The ARGT and ASF strategies significantly increased the quality of the GPC controller. The GPC+ARGT+ASF controller was effective for child patients with type 1 diabetes and can be potentially applied in the AP systems.

\section{Acknowledgements}

The authors would like to acknowledge that this study is supported by Guangdong Province Science and Technology (Project No. 2015A020214016), Science and Technology Program of Guangzhou (Project No. 201904010207), Young Innovative Talents Program in Universities and Colleges of Guangdong Province (Project No. 2018GkQNCX002) and GDHVPS 2016, as well as the funding support from Guangdong Province Medical Scientific Research Foundation (No. A2019142 and B2017093) and from the Research Grant of Guangdong Food and Drug Vocational College (No. 2018ZR028).

The authors declare no competing financial interests.

\section{References}

[1] Cho, N.H., et al., IDF Diabetes Atlas: Global estimates of diabetes prevalence for 2017 and projections for 2045 Diabetes Res Clin Pract, 2018. 138: 271-281.

[2] DiMeglio, L.A., C. Evans-Molina, and R.A. Oram, Type 1 diabetes. Lancet, 2018. 391(10138): 2449-2462.

[3] Gondhalekar, R., E. Dassau, and F.J. Doyle, 3rd, MPC Design for Rapid Pump-Attenuation and Expedited Hyperglycemia Response to Treat T1DM with an Artificial Pancreas. Proc Am Control Conf, 2014. 2014: 4224-4230.

[4] Turksoy, K., et al., Multivariable adaptive identification and control for artificial pancreas systems. IEEE Trans Biomed Eng, 2014. 61(3): 883-91.

[5] Blauw, H., et al., A Review of Safety and Design Requirements of the Artificial Pancreas. Ann Biomed Eng, 2016. 44(11): 3158-3172.

[6] Steil, G.M., Algorithms for a closed-loop artificial pancreas: the case for proportional-integral-derivative control. J Diabetes Sci Technol, 2013. 7(6): 1621-31.

[7] Huyett, L.M., et al., Design and Evaluation of a Robust PID Controller for a Fully Implantable Artificial Pancreas. Ind Eng Chem Res, 2015. 54(42): 10311-10321.

[8] Turksoy, K. and A. Cinar, Adaptive control of artificial pancreas systems - a review. J Healthc Eng, 2014. 5(1): $1-22$.

[9] Kamuran, T., et al., Multivariable adaptive closed-loop control of an artificial pancreas without meal and activity announcement. Diabetes Technology \& Therapeutics, 2013. 15(5): 386-400.

[10] Cameron, F., et al., A closed-loop artificial pancreas based on risk management. J Diabetes Sci Technol, 2011. 5(2): 368-79.

[11] Abbes, I.B., et al., A closed-loop artificial pancreas using a proportional integral derivative with double phase lead controller based on a new nonlinear model of glucose metabolism. J Diabetes Sci Technol, 2013. 7(3): 699-707.

[12] Soylu, S. and K. Danisman, In silico testing of optimized Fuzzy P + D controller for artificial pancreas. Biocybernetics \& Biomedical Engineering, 2018. 38(2).

[13] Clarke, D.W., E. Mosca, and R. Scattolini, Robustness of an adaptive predictive controller. Automatic Control IEEE Transactions on, 1994. 39(5): 1052-1056.

[14] Ioannou, P. and K. Tsakalis, A robust direct adaptive controller. IEEE Transactions on Automatic Control, 2003. 31(11): 1033-1043.

[15] Zhang, J., et al. Generalized predictive control for wind turbine systems. in Industrial Electronics and Applications. 2010.

[16] Liu, W., et al., Improved Generalized Predictive Control Algorithm for Blood Glucose Control of Type 1 Diabetes. Artif Organs, 2018. 
[17] Eren, M., Adaptive control strategy for regulation of blood glucose levels in patients with type 1 diabetes. Journal of Process Control, 2009. 19(8): 1333-1346.

[18] Man, C.D., et al., The UVA/PADOVA Type 1 Diabetes Simulator: New Features. J Diabetes Sci Technol, 2014. 8(1): 26-34.

[19] MohammadRidha, T., et al., Model Free iPID Control for Glycemia Regulation of Type-1 Diabetes. IEEE Trans Biomed Eng, 2018. 65(1): 199-206.

[20] Campos-Nã, Ã.e.E., J.E. Layne, and H.C. Zisser, In Silico Modeling of Minimal Effective Insulin Doses Using the UVA/PADOVA Type 1 Diabetes Simulator. J Diabetes Sci Technol, 2017: 193229681773534. 